\title{
Koisio Technology-Modulated Cell Culture Media can Lead to Markedly Increased Intracellular ATP Levels of Fibroblast Cell Cultures
}

\author{
Yinghui Men*, Mingchao Zhang*, Qi Zhu, Weihai Ying\# \\ ${ }^{1}$ Med-X Research Institute and School of Biomedical Engineering, Shanghai Jiao \\ Tong University, Shanghai 200030, P.R. China
}

* These two authors contributed equally to this work.

\#: Corresponding author

Weihai Ying, Ph.D.

Professor, School of Biomedical Engineering and Med-X Research Institute

Shanghai Jiao Tong University

1954 Huashan Road

Shanghai, 200030, P.R. China

E-mail: weihaiy@ @jtu.edu.cn

Running title: Koisio media can increase cellular energy 


\begin{abstract}
It has been reported that Koisio technology-modulated water without external additions of any substances can enter several types of aquaporins with significantly higher rate. In this study we determined the effects of Koisio technology-modulated cell culture media (Koisio media) on the energy state of cells. We found that for the lung fibroblast (L929) cell cultures, Koisio media without any additions of external substances led to approximately an 57\% increase in the intracellular ATP levels of the cell cultures at the Cell Passage 6 - 18, compared with regular cell culture media. Seemingly surprisingly, we did not find that Koisio media could significantly affect the mitochondrial membrane potential of the cells, compared with regular cell culture media. Collectively, our study has provided first evidence indicating that Koisio media can lead to remarkable increases in the intracellular ATP levels of the L929 cell cultures, suggesting significant potential of Koisio technology in generating energy-enhancing water. Our finding that Koisio media did not affect the mitochondrial membrane potential has suggested that novel mechanisms may underlie the effects of Koisio media on cellular energy.
\end{abstract}

Keywords: Water; ATP; Cellular energy; Mitochondrial membrane potential; Fibroblasts. 


\section{Introduction}

Intracellular ATP plays crucial roles in numerous cellular functions (Stryer, 1995). Therefore, maintenance of intracellular ATP levels is critical for cellular functions and cell survival (Almeida and Bolanos, 2001, Beal, 1992, Eguchi et al., 1997, Feldenberg et al., 1999, Stryer, 1995). Intracellular ATP in normal cells is generated mostly through mitochondrial oxidative phosphorylation, with a small portion of intracellular ATP being generated through glycolysis (Stryer, 1995). Intracellular ATP levels are regulated by numerous factors, failures of which can lead to a number of pathological changes (Almeida and Bolanos, 2001, Beal, 1992, Eguchi et al., 1997, Feldenberg et al., 1999, Stryer, 1995, Williams et al., 2015). Together with oxidative stress and inflammation, defects of energy metabolism belong to one of the most critical pathological factors of aging and numerous diseases (Beal, 1995, Ying, 1996a, 1996b, 1997a, 1997b).

It is highly valuable to discover novel and economic substances for enhancing the energy levels of human body. Since water drinking is required for human survival, it would be of great value if we can produce certain type of water with capacity of enhancing the energy levels of human body without addition of external substances. However, it is exceedingly difficult to reach this goal.

A previous study has reported that the ceramics produced by Koisio technology could produce water with increased permeability through aquaporins (Kozumi and Kitagawa, 2016). However, it has been unclear if Koisio technology-modulated solutions without additions of any external substances may affect intracellular ATP levels. In current study, we determined the effect of Koisio technology-modulated 
solutions on intracellular ATP levels, showing that Koisio media can produce remarkable increases in the intracellular ATP levels of fibroblast cell cultures.

\section{Materials and Methods}

\section{Cell Cultures}

L929 cells were purchased from the national collection of authenticated cell cultures (Shanghai, China). Cells were incubated in Dulbecco's modified Eagle medium (HyClone, Logan, UT, United States) supplemented with 10\% fetal bovine serum (Gibco, Carlsbad, CA, United States), 1\% $100 \mathrm{U} / \mathrm{ml}$ penicillin, and $100 \mu \mathrm{g} / \mathrm{ml}$ streptomycin at $37{ }^{\circ} \mathrm{C}$ in a humidified incubator under $5 \% \mathrm{CO}_{2}$.

\section{Modulations of cell culture media by Koisio technology}

Modulations of cell culture media were conducted by Koisio technology as reported previously (Kozumi and Kitagawa, 2016).

\section{Determinations of intracellular ATP levels}

As described previously (Zhang et al., 2018), cells in 24-well plates were harvested with $200 \mu \mathrm{L}$ Tris-EDTA buffer (0.1 M Tris-HCl, pH 7.75 and 1 mM EDTA) and heated at $95{ }^{\circ} \mathrm{C}$ for 5 minutes. For the determination of ATP, $25 \mu \mathrm{L}$ samples were 
mixed with $25 \mu \mathrm{L}$ PEP buffer $\left(5 \mathrm{mM}\right.$ PEP, $9 \mathrm{mM} \mathrm{MgCl}_{2}, 5 \mathrm{mM} \mathrm{KCl}$ in $0.1 \mathrm{M}$ TrisEDTA buffer, PH 7.75).

\section{Mitochondrial membrane potential assay}

As described previously (Zhang et al., 2018), mitochondrial membrane potential $\left(\Delta \psi_{\mathrm{m}}\right)$ was determined by flow cytometry-modulated JC-1 $\left(5,5^{\prime}, 6,6^{\prime}\right.$-tetrachloro1,1',3,3'-tetraethyl-benzimidazolylcarbocyanine iodide) assay according to the manufacturer's instruction. Cells were harvested by $0.25 \%$ trypsin-EDTA, and incubated in cell media containing $5 \mu \mathrm{g} / \mathrm{mL}$ JC-1 (Enzo Life Sciences, NY, USA) for 15 min at $37{ }^{\circ} \mathrm{C}$. After washed once with PBS, samples were analyzed by a flow cytometer (FACSAria II, BD Biosciences, NJ, USA) using the excitation wavelength of $488 \mathrm{~nm}$ and the emission wavelengths of $525 \mathrm{~nm}$ for green fluorescence, or the emission wavelengths of $575 \mathrm{~nm}$ for orange-red fluorescence. The $\Delta \psi_{\mathrm{m}}$ of each cell was calculated by the ratio of red fluorescence intensity to green fluorescence intensity. For each sample, the ratio of the cells with low $\Delta \psi_{\mathrm{m}}$ in total cell population was reported by the flow cytometer (FACSAria II, BD Biosciences, NJ, USA).

\section{Statistical analyses}

All data are presented as mean \pm SEM. Data were assessed by one-way ANOVA, followed by Student - Newman - Keuls post hoc test. $p$ values less than 0.05 were considered statistically significant. 


\section{Results}

Koisio media led to remarkable increases in the intracellular ATP levels of L929 cell cultures

We found that for the lung fibroblast L929 cell cultures, Koisio media led to approximately an 57\% increase in the intracellular ATP levels of the L929 cell cultures of the Cell Passage 6 - 18, compared with regular cell culture media (Fig. 1).

\section{Koisio media did not significantly affect the mitochondrial membrane potential of}

\section{L929 cell cultures}

Twenty-four hr after the cells were cultured in Koisio media or regular cell cultures, JC-1 assays were conducted. We did not find that Koisio media could significantly affect the mitochondrial membrane potential of L929 cell cultures, compared with regular cell culture media (Fig. 2).

We also conducted experiments using another protocol of cell treatment: Twenty-four hr after the cells were cultured in Koisio media or regular cell culture media, the cells were cultured in MEM for $1 \mathrm{hr}$. Subsequently, JC-1 assays were conducted. In the study using this protocol, Koisio media did not significantly affect the mitochondrial membrane potential of L929 cell cultures (Fig. 3).

\section{Discussion}

The major findings of our study include: First, Koisio media led to approximately an $57 \%$ increase in the intracellular ATP levels of the L929 cell cultures of the Cell 
Passage 6 - 18, compared with regular cell culture media; and second, Koisio media did not significantly affect the mitochondrial membrane potential of the cell cultures, compared with regular cell culture media.

It is highly valuable to discover novel and economic substances for enhancing energy levels of human body. Since water drinking is required for human survival, it would be of great value if we can produce certain type of water with capacity of enhancing energy levels without addition of external substances. Our findings have shown that Koisio technology-modulated solutions have significant capacity to increase intracellular ATP levels. This finding has suggested that Koisio technology-modulated water could have significant capacity to increase intracellular ATP levels.

It is noteworthy that Koisio technology modulates water or solutions solely by physical modulations without additions of any external substances. To our knowledge, our finding that Koisio technology-modulated solutions have significant capacity to increase intracellular ATP levels is the first report that solutions modulated solely by physical approaches can have the capacity of increasing intracellular ATP levels. Future studies are needed to investigate the mechanisms underlying this novel finding.

Our study has provided first evidence indicating that Koisio media led to remarkable increases in the intracellular ATP levels of the L929 cell cultures without affecting mitochondrial membrane potential. This observation is seemingly surprising, considering the fact that mitochondrial oxidative phosphorylation is causative to the cellular generation of a large majority of ATP. These findings have suggested that Koisio media can lead to remarkable increases in intracellular ATP levels by novel energy-enhancing mechanisms. It is warranted to conduct future studies to investigate the novel mechanisms. 


\section{References:}

Almeida A, Bolanos JP. A transient inhibition of mitochondrial ATP synthesis by nitric oxide synthase activation triggered apoptosis in primary cortical neurons. $\mathbf{J}$ Neurochem 2001;77(2):676-90.

Beal MF. Does impairment of energy metabolism result in excitotoxic neuronal death in neurodegenerative illnesses? Ann Neurol 1992;31(2):119-30.

Beal MF. Aging, energy, and oxidative stress in neurodegenerative diseases. Ann Neurol 1995;38(3):357-66.

Eguchi Y, Shimizu S, Tsujimoto Y. Intracellular ATP levels determine cell death fate by apoptosis or necrosis. Cancer Res 1997;57(10):1835-40.

Feldenberg LR, Thevananther S, del Rio M, de Leon M, Devarajan P. Partial ATP depletion induces Fas- and caspase-mediated apoptosis in MDCK cells. Am J Physiol 1999;276(6 Pt 2):F837-46.

Kozumi T, Kitagawa Y. Water structure changes induced by ceramics can be detected by increased permeability through aquaporin. Biochem Biophys Rep 2016;5:353-8.

Stryer L. Biochemistry. New York: W.H. Freeman and Company, 1995.

Williams GS, Boyman L, Lederer WJ. Mitochondrial calcium and the regulation of metabolism in the heart. J Mol Cell Cardiol 2015;78:35-45.

Ying W. Deleterious network hypothesis of Alzheimer's disease. Med Hypotheses 1996a;46(5):421-8.

Ying W. A new hypothesis of neurodegenerative diseases: the deleterious network hypothesis. Med Hypotheses 1996b;47(4):307-13.

Ying W. Deleterious network hypothesis of aging. Med Hypotheses 1997a;48(2):143-

8. 
Ying W. Deleterious network: a testable pathogenetic concept of Alzheimer's disease. Gerontology 1997b;43(4):242-53.

Zhang J, Wang C, Shi H, Wu D, Ying W. Extracellular Degradation Into Adenosine and the Activities of Adenosine Kinase and AMPK Mediate Extracellular NAD ${ }^{+}$-Produced Increases in the Adenylate Pool of BV2 Microglia Under Basal Conditions. Front Cell Neurosci 2018;12:343. 


\section{Figure Legends:}

Fig. 1. Koisio media (KM) led to remarkable increases in the intracellular ATP levels of L929 cell cultures. Cultures of the cells in Koisio media led to an approximately 57\% increase in the intracellular ATP levels of the L929 cell cultures of the Cell Passage 6-18, compared with the cells cultured in regular cell culture media. The ATP assays were conducted 24 - 36 hrs after the cells were plated. $\mathrm{N}=34$. \#\#\#, $P$ $<0.001$ (Unpaired t-test).

Fig. 2. Koisio media (KM) did not significantly affect the mitochondrial membrane potential of the $\mathbf{L 9 2 9}$ cell cultures. Koisio media did not significantly affect the mitochondrial membrane potential of the L929 cell cultures of the Cell Passage 6 - 18, compared with the cell cultures cultured in regular media. The JC-1 assays were conducted $24-36$ hrs after the cells were plated. $\mathrm{N}=7$.

\section{Fig. 3. Koisio media (KM) did not significantly affect the mitochondrial membrane} potential of the $\mathbf{L 9 2 9}$ cell cultures in a different cell culture protocol. Koisio media did not significantly affect the mitochondrial membrane potential of L929 cell cultures of the Cell Passage 6 - 18, compared with the cell cultures cultured in regular cell culture media. Twenty-four hr after the cells were cultured in Koisio media or regular cell culture media, the cells were cultured in MEM for $1 \mathrm{hr}$. Subsequently, JC-1 assays were conducted. $\mathrm{N}=6$. 


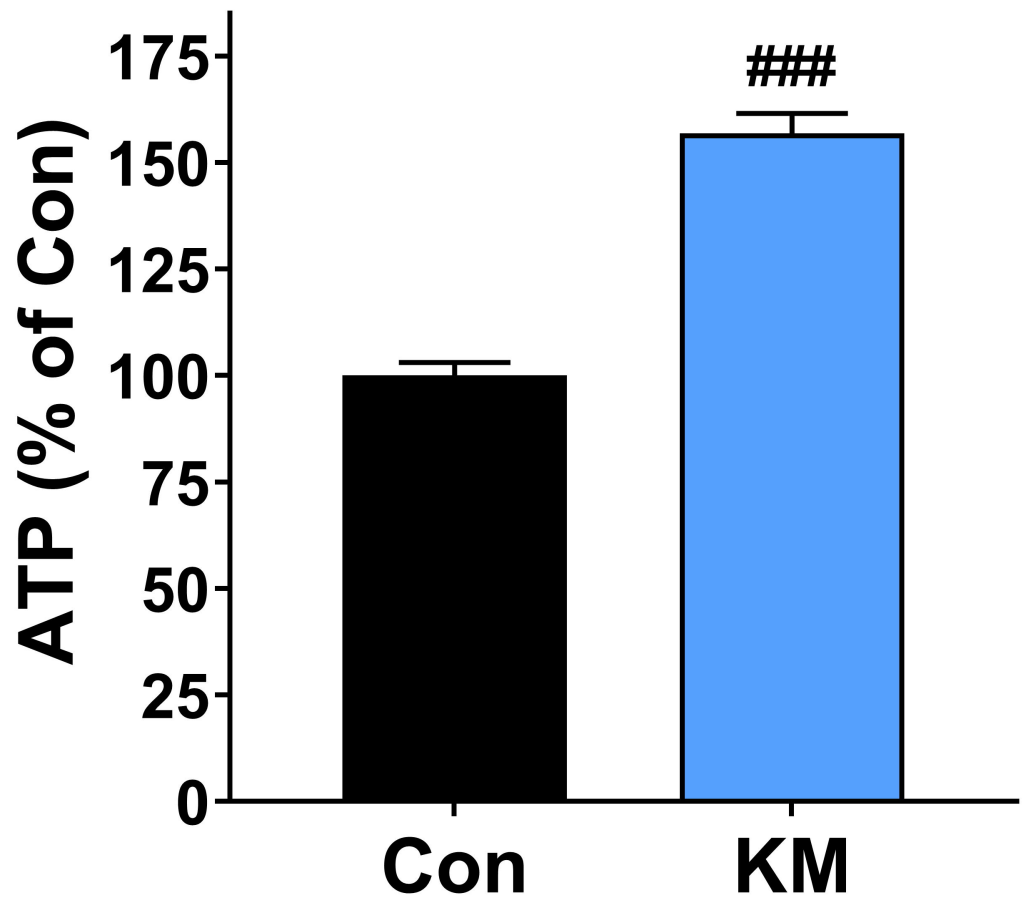


A

\section{Con}
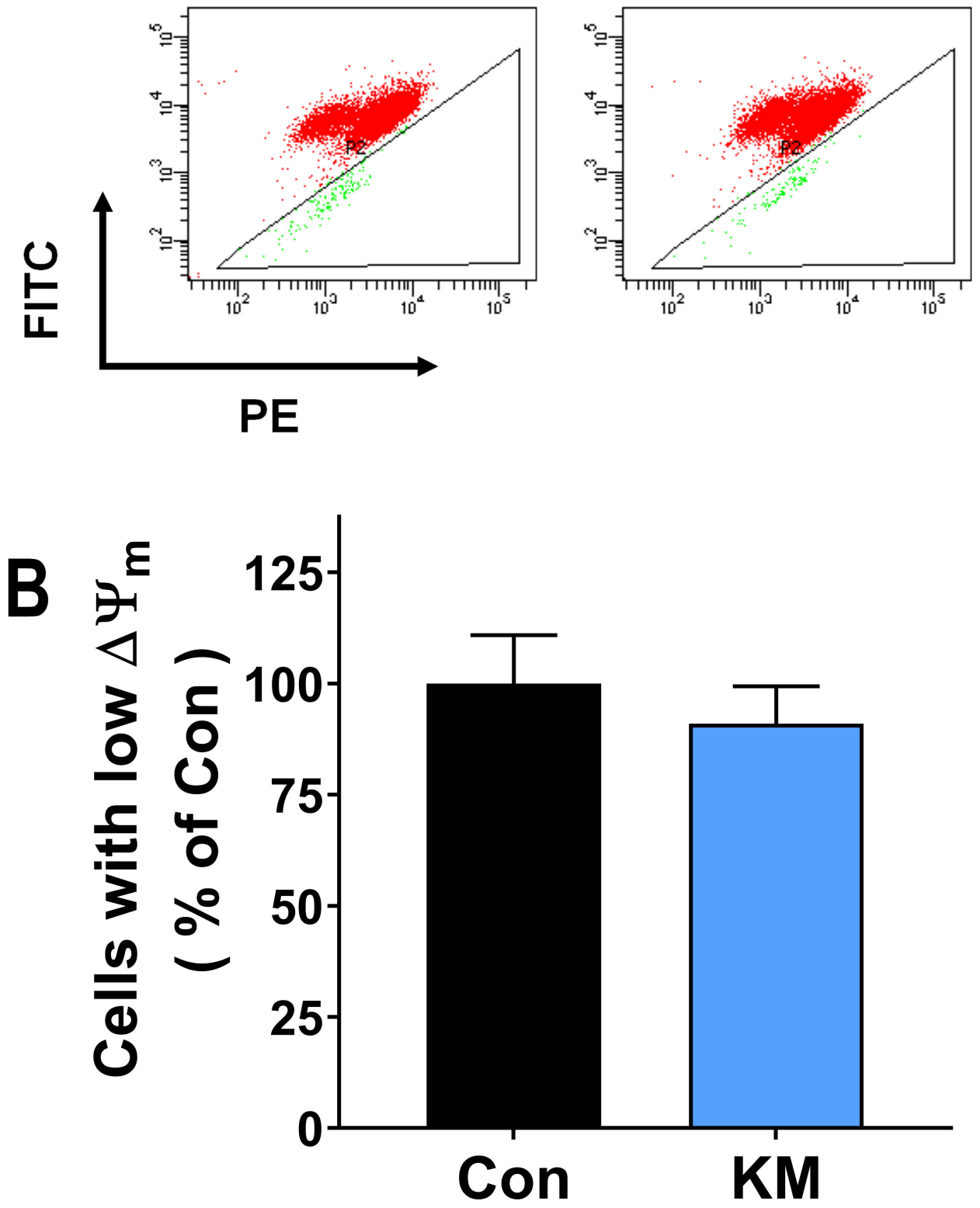
A

Con
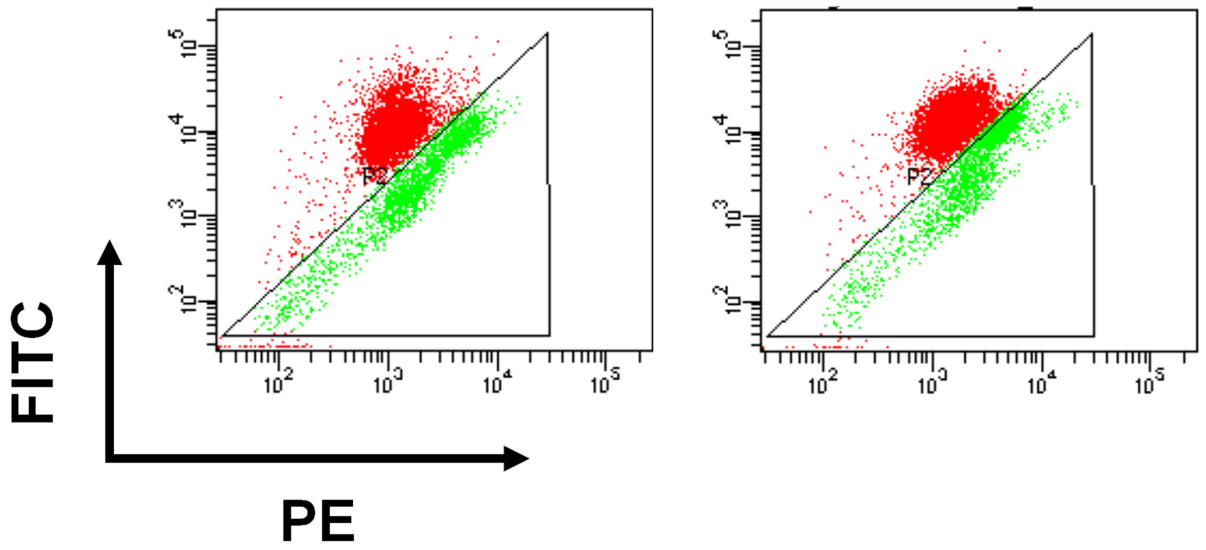

B

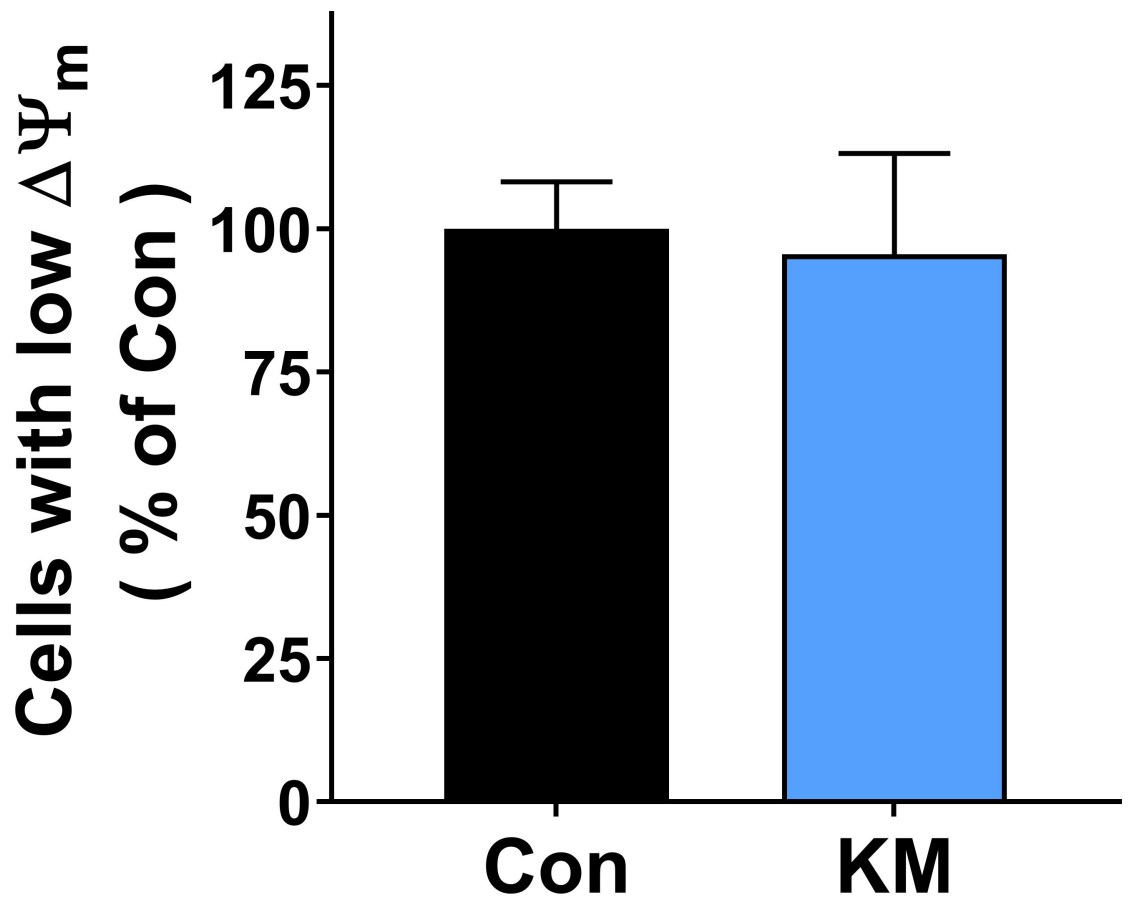

